\title{
New process results in smoother indium antimonide substrates
}

Patrick Flint, Lisa P. Allen, Gordon Dallas, Daniel Bakken, Kevin Blanchat, Shiva R. Vangala, William Goodhue, David Bliss, and Helen Dauplais

Modified chemomechanical polishing can reduce five-fold the surface roughness of large-diameter indium antimonide substrates used in advanced imaging systems and devices.

The high optical transparency of indium antimonide (InSb) substrates makes them attractive for IR focal-plane arrays (IRFPAs) and detectors, free-space communications, transistors, and integrated optoelectronics. ${ }^{1-3}$ A large-diameter crystalline InSb surface can accommodate new and bigger advanced IRFPA designs. However, the $150 \mathrm{~mm}$ diameter of these antimonides poses unique surface-polishing challenges to substrate manufacturers. To improve the resolution and sensitivity of highperformance IRFPA imaging systems in the $1-5.4 \mu \mathrm{m}$ region $\left(77^{\circ} \mathrm{K}\right)$, the substrates' surface must meet or surpass stringent demands. In particular, for detector-fabrication processes requiring epitaxy growth for the device layer, the starting InSb surface must be ultrasmooth, less than one atomic layer in roughness. It should also desorb the $\mathrm{Sb}$ and In surface oxides in an abrupt and fast outgassing process. ${ }^{4}$ A consistent and easily desorbable surface oxide helps improve advanced-device epilayer yield and performance in Sb-based IRFPA manufacturing.

The substrate's chemomechanical-polish (CMP) history is a primary factor in InSb surface smoothness and oxide desorption. We wanted to consistently produce surfaces suitable for recent epitaxy processes with stringent substrate-surface specifications. To do so, we analyzed the substrate-surface orientation, atomic roughness, oxide desorption, and crystallinity as a function of CMP process. Our resulting CMP process for largerdiameter InSb single-crystal surfaces has produced a smoother InSb starting surface and abrupt oxide-desorption characteristics suitable for device-layer molecular-beam-epitaxy (MBE) material growth. ${ }^{5}$

We applied a standard semiconductor-grade CMP to two groups of antimonide substrates separated from the same boule. We used a proprietary CMP process for group A. For group

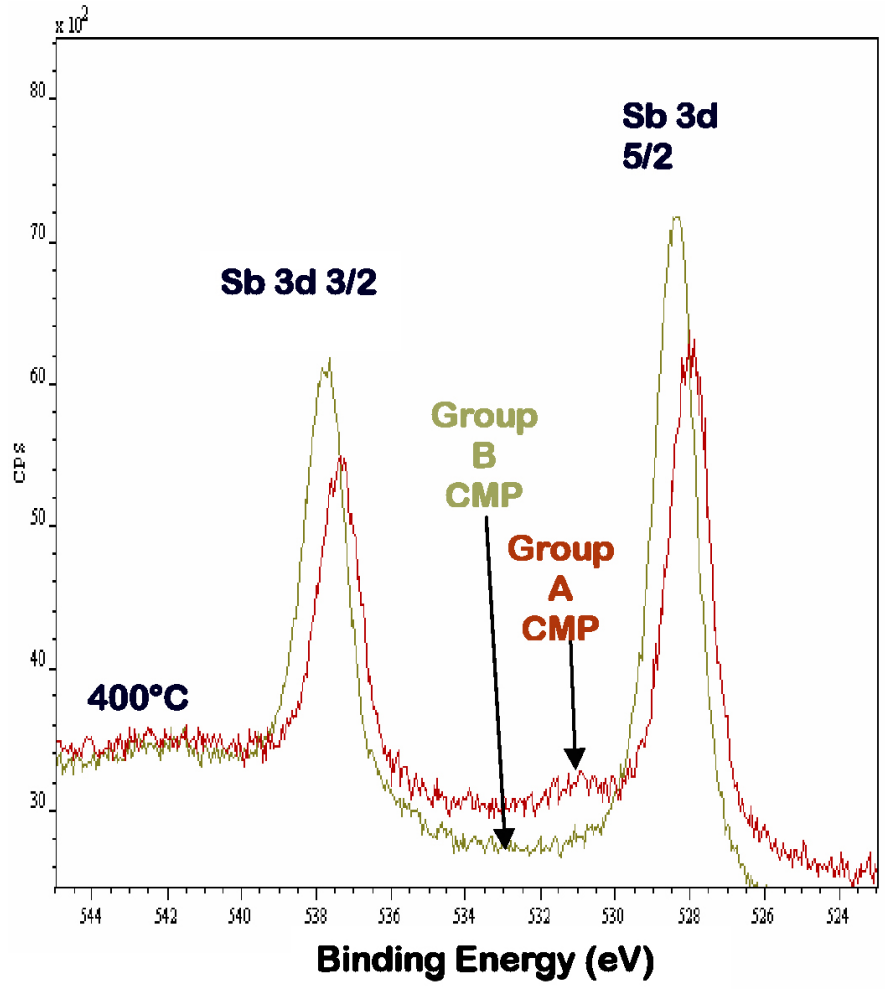

Figure 1. Thermal $x$-ray photoelectron spectroscopy analysis spectra in electron volts $(\mathrm{eV})$ for the antimonide $(\mathrm{Sb}) 3 d$ (electron orbital) binding-energy peaks of both Group $A$ and B chemomechanical polish surfaces at $400^{\circ} \mathrm{C}$ shows the Sb oxide removed for Group B polished surfaces. CMP: Chemomechanical polish.

B, we used a process with the same constituents but with a new differential ratio between $\mathrm{pH}$ (a measure of the acidity or basicity of a solution) and oxidizer and buffering percentages. Because they were adjacent slices from the same boule, the experiment varied only the polishing constituent ratio. We compared the surfaces by atomic-force microscopy (AFM), Hall-effect doping-concentration analysis, glancing-angle $x$-ray diffraction (XRD), thermal x-ray photoelectron spectroscopy 
(TXPS) analysis of surface desorption as a function of temperature, and MBE growth using reflection high-energy electrondiffraction (RHEED) surface-reconstruction patterns.

We found that the group A standard polish retained a distinct presence of residual In and $\mathrm{Sb}$ oxides when the InSb surface was heated to $400^{\circ} \mathrm{C}$. This is evidenced by the associated oxide peaks of the $\mathrm{Sb} 3 \mathrm{~d} 3 / 2$ (electron orbitals) and In $3 \mathrm{~d} 3 / 2$ TXPS spectra as a function of temperature (from room temperature to $450^{\circ} \mathrm{C}$ ). The TXPS data for the group B polish showed that both the $\mathrm{Sb} 3 \mathrm{~d} 3 / 2$ and In $3 \mathrm{~d} 3 / 2$ binding-energy peaks completely narrowed, and the associated oxide peaks were absent by $400^{\circ} \mathrm{C}$. Figure 1 shows the $\mathrm{Sb}$ binding-energy peak comparison. InSb homoepitaxy (MBE and RHEED) results also indicate that group B InSb CMP substrates show an abrupt and desirable $1 \times 1$ surface-reconstruction pattern by $430^{\circ} \mathrm{C}$ in the low-pressure MBE outgassing stage.

Glancing-angle XRD and Hall effect revealed that the crystallinity for group A and B substrates were inherently the same and precisely aligned at $\operatorname{InSb}(111) \mathrm{B} \pm 0.01^{\circ}$. The final epiroughness was significantly higher for group A surfaces than for group B. Typically, as measured by $100 \mu \mathrm{m}^{2}$ AFM-area images, the epiatomic roughness $\left(\mathrm{R}_{\mathrm{a}}\right)$ was $4.7 \mathrm{~nm}$, and the peak-to-valley morphology measurements were as high as $84.5 \mathrm{~nm}$ for the group A surfaces. More significantly, we did not observe any episurface steps or terraces. The surface morphology for group B substrates consisted of clear episteps and terraces with an average $R_{a}$ of $0.73 \mathrm{~nm}$ and peak-to-valley heights of $10.7 \mathrm{~nm}$.

Various InSb surface-material evaluations of the two CMP processes indicated that group B surfaces allowed a lower temperature and more abrupt surface-oxide-desorption process for epigrowth. The improved low-thermal desorbtion character provided a smooth template for the resulting steps-and-terrace morphology of the InSb episurface.

In summary, the new differential ratio between the $\mathrm{pH}$, the oxidizer percentage, and the buffering percentage of the group $B$ etching solution for InSb surface-oxide removal is effective for InSb(111)B epigrowth surfaces. With a highly desorbable interface, the new InSb CMP finish may provide more widespread adaptation of the crystal for low-power quantum-well-depletion transistors, advanced large-diameter IRFPAs, and optoelectronic applications. We will continue to research improved polishing compounds and processes. We also are working to develop new specifications for larger-diameter substrates.

We thank all engineers and technicians at Galaxy Compound Semiconductors Inc. for their hard work and devotion to the antimonide crystal-growth and fabrication process. We gratefully acknowledge the US Department of Defense (contract FA8718-07-C-0035) for support.

\section{Author Information}

Patrick Flint, Lisa P. Allen, Gordon Dallas, Daniel Bakken, and Kevin Blanchat

Galaxy Compound Semiconductors Inc.

Spokane, WA

Patrick Flint, operations manager, has more than 17 years of semiconductor engineering experience in manufacturingimprovement programs. He received the 2004 Honeywell Chairman's Award, the Chairman's Award Grand Prize, and the 2005 Quest for Excellence Value Creation Award. He graduated with multiple degrees in chemistry and physics from Washington State University (1994), and has numerous publications pertaining to gallium antimonide ( $\mathrm{GaSb}$ ) and indium antimonide ( $\mathrm{InSb}$ ) crystal growth and characterization.

Lisa Allen, technology programs director, has more than 20 years of experience in semiconductor materials research and development. A materials-engineering graduate of the Massachusetts Institute of Technology (MIT) and the University of California at Berkeley, she was the general chair of the 1998 IEEE International Silicon-on-Insulator conference and an executive committee member of the IEEE Nuclear and Space Radiation Effects conference. With more than 120 publications and five patents, she is regional vice president of the MIT Educational Council and a senior member of the IEEE and The Minerals, Metals, \& Materials Society.

Gordon Dallas, co-founder, has extensive GaSb and InSb crystalgrowth experience. He previously worked at Johnson-Matthey Electronics as a crystal-growth engineer responsible for compound semiconductor crystals for IR detectors. In 1999, he spun off the Johnson-Matthey III-V Substrate unit as Galaxy Compound Semiconductors. Gordon has co-authored numerous antimonide-material conference and journal publications.

Daniel Bakken, co-founder, received his BS in physics/astrophysics from Eastern Washington University in 1990. He previously worked at Johnson-Matthey Electronics as a crystalgrowth engineer responsible for compound semiconductor crystals for IR detectors. In 1999, he spun off the Johnson-Matthey III-V Substrate unit as Galaxy Compound Semiconductors. He has authored numerous antimonide publications. 
Shiva R. Vangala and William Goodhue

Photonics Center

University of Massachusetts

Lowell, MA

Shiva Vangala is a postdoctoral research fellow whose research interests include MBE growth and characterization of compound semiconductor structures for optoelectronic and photonic devices such as long-wavelength detectors and quantum-well lasers. He received MS and PhD degrees from the Department of Physics and Applied Physics, University of Massachusetts at Lowell. He is a member of American Physical Society and SPIE.

William Goodhue is a professor in the Department of Physics and a staff member of MIT's Lincoln Laboratory. His research interests include quantum-well-device physics, photonic and optoelectronic device fabrication, terahertz quantum-cascade lasers, nanosphere lithography applied to quantum-dot technology, and MBE. His work has been reported in numerous articles, reports, book chapters, and meeting speeches. He is a senior member of IEEE.

\section{David Bliss and Helen Dauplais}

Sensors Directorate

Air Force Research Laboratory

Hanscom Air Force Base, MA
References

1. P. C. Klipstein, E. Harush, E. Jacobsohn, O. Klin, T. Markovitz, E. Saguy, J. Oiknine-Schlesinger, I. Shtrichman, M. Yassen, and E. Weiss, High-performance InAlSb MWIR detectors operating at 100K and beyond, Proc. SPIE 6206, p. 62060, 2007 2. T. Ashley, A. R. Barnes, L. Buckle, S. Datta, A. B. Dean, Emeny, T. Fearn, D. G. Hayes, K. P. Hilton, R. Jefferies, T. Martin, K. J. Nash, T. J. Phillips, W. H. A. Tang, and R. Chau, InSb-based quantum well transistors for high-speed, low-power applications. http://www.gaasmantech.org/Digests/2005/2005papers/14.18.pdf. Accessed 3 August 2009.

3. C. J. Hill, A. Soibel, S. A. Keo, J. M. Mumolo, D. Z. Ting, S. D. Gunapala, D. R. Rhiger, R. E. Kvaas, and S. F. Harris, Demonstration of mid-and longwavelength infrared antimonide-based focal plane arrays, Proc. SPIE 7298, p. 729804, 2009. doi: $10.1117 / 12.818692$

4. O. Klin, S. Grossman, N. Snapi, M. Brumer, I. Lukomsky, M. Yassen, B. Yofis, A. Glozman, A. Zemel, T. Fishman, E. Berkowicz, O. Magen, J. OiknineSchlesinger, I. Shtrichman, E. Weiss, and P. C. Klipstein, Progress with antimonidebased detectors at SCD, Proc. SPIE 7298, p. 72980, 2009.

5. S. R. Vangala, X. Qian, M. Grzesik, C. Santeufemio, L. P. Allen, G. Dallas, H. Daupliase, K. Vaccaro, S. Q. Wan, D. Bliss, and W. D. Goodhue, MBE growth of homoepitaxial layers on CMP processed InSb(100) and InSb(111)B substrates, J. Vac. Sci. Technol. B 24 (3), pp. 1634-1638, 2006. 